\title{
West syndrome with hyperkinesia and cortical visual impairment: A case report of GRIN1 encephalopathy
}

\author{
Seul A Choi ${ }^{\oplus}$ and Young Ok Kim*® \\ Department of Pediatrics, Chonnam National University Children's Hospital, Chonnam National University Medical School, Gwangju, Korea
}

\begin{abstract}
West syndrome (WS) presenting with infantile spasms, developmental delay, and hypsarrhythmia has genetic etiology in some patients. Movement disorders or visual impairment that share genetic underpinnings with infantile spasms can provide diagnostic clues for specific genetic mutations. Mutations of the GRIN1 gene encoding the glutamate receptor inotropic Nmethyl-D-aspartate subunit can result in WS with hyperkinetic movements, cortical visual impairment, autistic features, and bilateral polymicrogyria. An 11-month-old boy with WS showed hyperkinetic movements and visual impairment. Brain magnetic resonance imaging and metabolic investigations revealed no abnormalities. Whole-exome sequencing revealed a novel likely pathogenic variant (c.1561_1563del; p.Asn521del) of GRIN1 (NM_007327.3). The proband was treated with vigabatrin and became seizure-free within one week. Notably, the cortical blindness improved within 3 months and the hyperkinetic movements resolved one year after the proband became seizure-free. To the best of our knowledge, this is the first report of GRIN1 encephalopathy in Koreans.
\end{abstract}

Key words: Glutamate receptors, Infantile spasms, Hyperkinesis, Blindness.

\section{Introduction}

West syndrome (WS) is one of the most well-known childhood epilepsy syndromes; it presents with a triad of infantile spasms in clusters, developmental delay, and typical hypsarrhythmia on interictal electroencephalogram (EEG) [1]. With the generally increasing use of next-generation sequencing as a diagnostic tool in epilepsy, genetic etiologies have been demonstrated in WS patients. Several genes have been reported, including those encoding aristaless-related homeobox, $\mathrm{X}$-linked aristaless (ARX), the cyclin-dependent kinase-like 5 (CDKL5), and syntaxin-binding protein 1 (STXBP1) [1-4]. Some WS patients with specific genetic mutations (e.g., STXBP1) frequently display movement disorders (e.g., dyskinesia, tremor, and stereotypy) that need to be differentiated from spasms [4].

Mutations of the gene encoding the glutamate receptor ionotropic N-methyl-D-aspartate (NMDA) subunit 1 (GRIN1) result in neurodevelopmental disorders with or without hyperkinetic movements and seizures (NDHMS) [5-15]. NMDA receptors are ligand-gated ion channels composed of two glycinebinding subunits (GluN1) and two glutamate-binding subunits; the GluN1 subunit is encoded by GRIN1 [5-11,14,15]. GluN1 is ubiquitous in the brain during all stages of development [14]. All individuals with GRIN1-NDHMS demonstrate developmental delay from early infancy and have a non-syndromic intellectual disability [5-13]. In addition to epileptic seizures, abnormal movement disorders and cortical visual impairment have been reported to be characteristic in patients with GRIN1 encepha-

Received: 7 April 2021, Revised: 9 May 2021, Accepted: 14 May 2021, Published: 30 June 2021

${ }^{*}$ Corresponding author: Young Ok Kim, M.D., Ph.D. iD https://orcid.org/0000-0002-7873-1140

Department of Pediatrics, Chonnam National University Childrens' Hospital, 42 Jebong-ro, Dong-gu, Gwangju 61469, Korea.

Tel: +82-62-220-6646, Fax: +82-62-222-6103, E-mail: ik052@jnu.ac.kr

Conflict of interest: The authors declare that they do not have any conflicts of interest.

(C) This is an open-access article distributed under the terms of the Creative Commons Attribution Non-Commercial License (http://creativecommons.org/licenses/by-nc/4.0/) which permits unrestricted non-commercial use, distribution, and reproduction in any medium, provided the original work is properly cited.

(c) Copyright 2021 by the Korean Society of Medical Genetics and Genomics 
lopathy, although their prognoses have not been determined yet $[5-14,16]$. Some patients with GRIN1 mutations demonstrate autistic features and polymicrogyria $[9,11,12]$.

Using whole-exome sequencing (WES), we found a novel mutation of GRIN1 (c.1561_1563del; p.Asn521del) in a boy with infantile spasms, hyperkinetic movements, and cortical visual impairment. Notably, his spasms were resolved after one week of treatment with vigabatrin, and his cortical blindness disappeared completely within three months. In addition, his hyperkinetic movements were ameliorated; they were almost absent one year after the proband became seizure-free. His development has improved slowly.

This study was approved by the Human Research Ethics Committee of the Chonnam National University Hospital (IRB number, CNUH-2017-167). The Chonnam National University Hospital Biomedical Research Institute Biobank provided the biospecimens after approval from the review board. Informed consent was obtained from the parents of the proband.

\section{Case}

An 11-month-old boy presented with unpleasant, brief, and stiffly bending of his body that initially appeared intermittently at seven months of age and reappeared recurrently two weeks before his visit to our hospital; his condition was diagnosed as tonic spasms. He was born uneventfully to healthy parents at a gestational age of 38 weeks, and he weighed $2.87 \mathrm{~kg}$. He visited another hospital at eight months of age due to global developmental delay and axial hypotonia without eye contact or head control. At that time, his hyperkinetic and unpatterned movements that aggravated gradually were reported by his parents, although their onset was not definitively determined. The brain magnetic resonance imaging (MRI) findings in that hospital were normal. When he was nine months of age, almost all the subsets in the Bayley Scales of Infant Development ( $3^{\text {rd }}$ Edition) were below 16 days of age, except gross motor development estimated at 3 months and 20 days of age.

During his first visit to our hospital, his weight was $9.2 \mathrm{~kg}$ (25th-50th percentile), his height was $77.6 \mathrm{~cm}$ (75th-90th percentile), and the head circumference measured $43.7 \mathrm{~cm}$ (10th percentile). He could not stare at, or follow, moving objects; however, he was able to fixate on light intermittently. He could not sit alone, although he could flip and hold an object in his hand for a while. He could not vocalize words such as "mama" or "papa." However, he turned his head toward sounds. An EEG analysis revealed high-voltage, dysmorphic, and slow delta/ theta activities with posteriorly-dominant multifocal sharps (hypsarrhythmia; Fig. 1). He was treated with vigabatrin (50 mg/ $\mathrm{kg} /$ day for the first 3 days and then $75 \mathrm{mg} / \mathrm{kg} /$ day) and became seizure-free within one week. Blood amino acid and urine organic acid analyses, chromosomal analysis, and a chromosomal microarray study showed no abnormal results. Abdominal ultrasonography revealed no anomalies in the internal organs.

His vision and development gradually improved after he became seizure-free after treatment with vigabatrin. Three months later, he was able to sit alone, make eye contact, and watch and follow objects moving at angles of more than 180 degrees. However, dyskinetic movements in his trunk and limbs persisted.

At 17 months of age, a visual evoked potential test was performed yielding normal results. His hyperkinetic movements were markedly ameliorated, enabling him to stand alone. At 23 months of age, vigabatrin was discontinued. His hyperkinetic

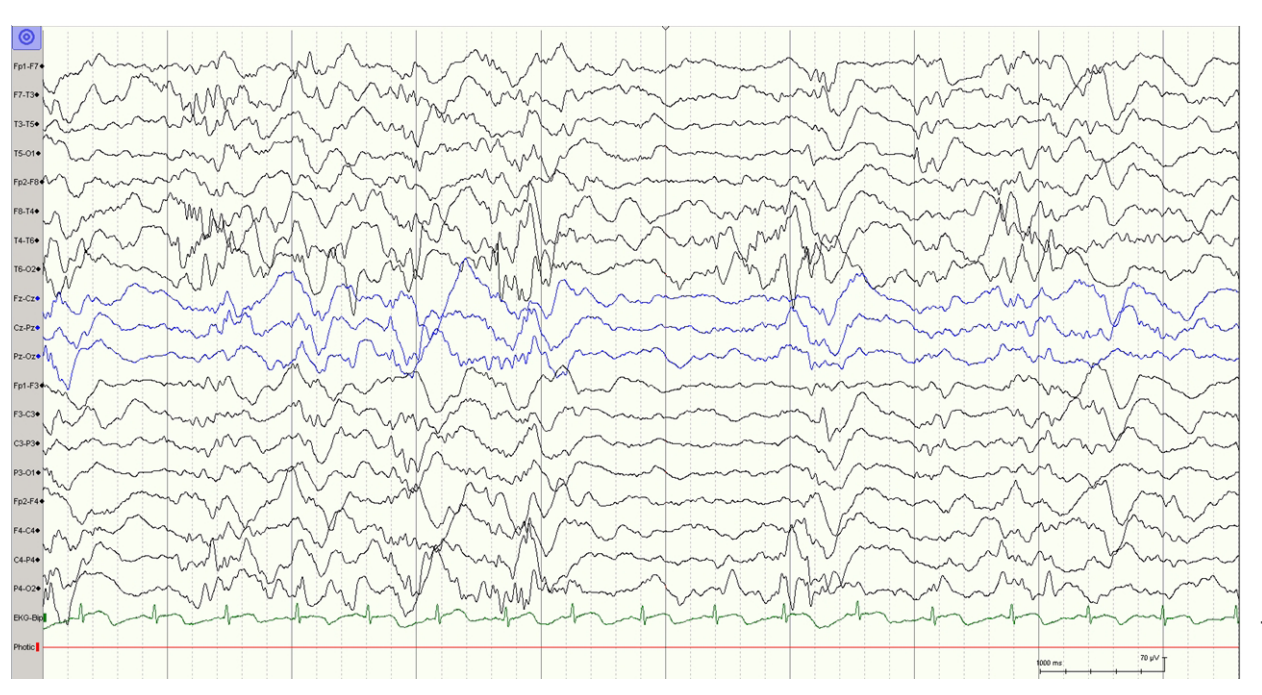

Fig. 1. Electroencephalogram (EEG) of the patient obtained during admission at our hospital. This interictal sleep EEG shows high-voltage, dysmorphic, and slow delta/ theta activities with posteriorly-dominant multifocal sharps (hypsarrhythmia). 
movements were minimized. Although his fine motor, adaptive, language, and social functions still lagged at 12 months of age, at 26 months of age, he was able to stiffly run and ascend the stairs with one hand on the railing.

WES using the Sure Select Human All Exon V5 (Agilent Technologies Inc., Santa Clara, CA, USA) was performed in the proband; the NextSeq 500 System (Illumina Inc., San Diego, CA, USA) was used. The reference databases of single-nucleotide polymorphisms used to prioritize the variants included the Genome Aggregation Database, the Exome Aggregation Consortium (Exac), and the Korean Reference Genome Database (http:// coda.nih.go.kr/coda/KRGDB/index.jsp). The WES analysis revealed the likely pathogenic heterozygous mutation (c.1561_1563del; p.Asn521del) of GRIN1 (NM_007327.3), which was verified using direct sequencing (Fig. 2). This novel mutation was de novo, as it was not found in his parents. This variant has not been reported in any human genome database.

\section{Discussion}

The hyperkinetic movements in our proband with WS initially suggested STXBP1 encephalopathy, as his epileptic spasms were responsive to vigabatrin. However, cortical visual impairment is a rare phenotype in patients with STXBP1 encephalopathy. In this proband, a GRIN1 mutation (c.1561_1563del; p.Asn521del) was discovered; GRIN1 is one of the causative genes of cortical blindness [16]. This mutation is novel, and to the best of our knowledge, this is the first time it was reported in Koreans.
GRIN1 has different domains: the amino-terminal, first and second ligand-binding (S1 and S2), 4 transmembrane (M1 to M4), calmodulin-binding, and carboxyl-terminal domains $[6,7,11,14]$. Most GRIN1 mutations cluster within, or proximally to, the transmembrane domains [7]. The novel mutation of p.Asn521del in our proband was located in S1, with phenotypes that did not seem severe. Concerning the genotype-phenotype correlation, the GRIN1 mutations in the patients with extensive bilateral polymicrogyria have been found to be located in $\mathrm{M} 3$ or S2, with the patients exhibiting severe phenotypes, including intractable epilepsy, cortical blindness, microcephaly, profound delay, and spastic tetraplegia $[11,12]$. Heterozygous de novo mutations have been reported in most patients with GRIN1NDHMS; however, homozygous mutations have also been detected in some patients [5-14]. Most GRIN1 mutations result in a loss of function [5-15].

The hyperkinetic movements in our proband were gradually ameliorated after the seizures subsided, and they were minimal at 23 months of age. In a single case reported by Pironiti et al. [13], abnormal movements were observed evidently during infancy and less prominently at five years of age. Data regarding the prognosis of movement disorders are limited. Lemke et al. [7] reported dyskinesia/dystonia/choreatic movements in 61\%, and oculogyric crisis in 22\% of total 23 patients with GRIN1NDHMS, and stereotyped movements in 33\% (of total 21 patients with data).

Cortical visual impairment in our proband could not be prognosticated initially, although a favorable visual response was ob-

\section{Father}

IGATCGTGGCGCCGCTAACCATAAACAACGACGCGCGCAGTACATCGAGITI

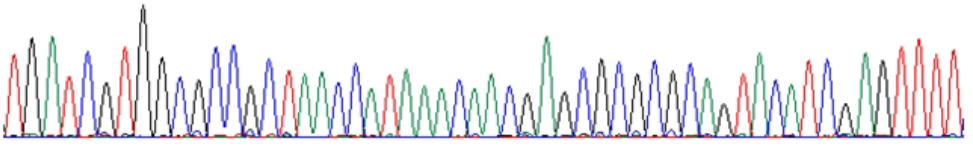

Mother

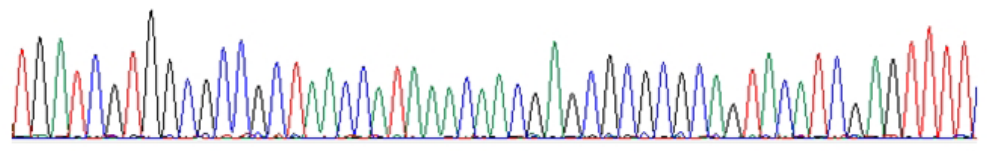

\section{Proband}

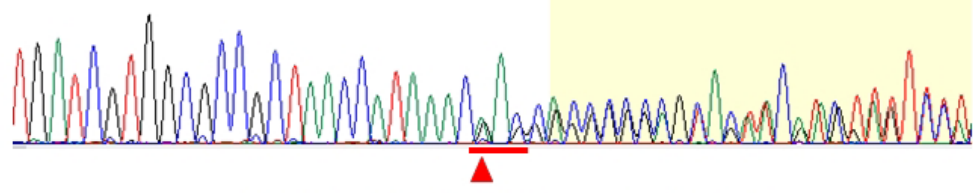

GRIN1 (c.1561_1563del; p.Asn521del)
Fig. 2. Electropherogram of the proband's family. It shows a de novo heterozygous in-frame deletion (c.1561_1563del; p.Asn521del) of GRIN1 (NM_007327.3) only in the proband but a wild-type sequence in his parents. 
served within three months after his seizures disappeared. Nevertheless, in a report by Pironti et al. [13], a girl with intractable seizures was poorly reactive to visual stimuli even at 5 years of age. Data regarding visual improvement in GRIN1-NDHMS are still lacking. As stated earlier, cortical visual impairment was reported in 22\% of 23 patients with GRIN1-NDHMS in a study by Lemke et al. [7]. Delayed visual fixation and problems with face recognition have to be closely observed in young infants with GRIN1-NDHMS to prevent missing their visual defect [16].

Seizures in our proband were only characterized by infantile spasms responsive to vigabatrin. However, seizures in GRIN1NDHMS are of different types (e.g., spasms, tonic, tonic-clonic, atonic, or myoclonic seizures) and have varying onsets $(<4$ weeks to 11 years of age) and drug responsiveness [5-14]. In a report by Lemke et al. [7], 70\% of the affected patients experienced seizures. On EEG analysis, hypsarrhythmia, focal or multifocal spikes/sharps, continuous slow waves with focal discharges, or generalized epileptiform discharges were recorded [7]. The brain MRI was normal in most patients with GRIN1-NDHMS, whereas extensive bilateral polymicrogyria, brain atrophy, and ventriculomegaly and a thin corpus callosum were reported in others $[6-11,13]$.

The global developmental delay in our proband was as severe as that observed in most patients with GRIN1 mutations [514]. Axial hypotonia was apparent in our proband; in a study by Lemke et al. [7], it showed a prevalence of 71\%. Although spasticity and feeding difficulties were not observed in our proband, they have been reported in 29\% and 39\% of patients with GRIN1-NDHMS, respectively [7]. Autistic spectrum disorder (35\%), sleep disorders (35\%), and aggression with self-injurious behaviors (17\%) were reported in some patients [7]. The facial gestalt in our proband was not dysmorphic, although some patients have non-specific features such as deep-set eyes, an elongated face, and a pointed chin [6-8].

In the present case, a rare genetic abnormality in WS, a GRIN1 mutation, was detected in a boy with hyperkinesia and cortical visual impairment; this novel mutation was located within the S1 domain. The patient showed a favorable prognosis as his symptoms improved, unlike what has been reported in previous cases with intractable epilepsy. The spasms disappeared within a week after treatment with vigabatrin. His cortical blindness disappeared completely within three months, and his hyperkinetic movements were almost resolved after one year. His development has improved slowly.

\section{Acknowledgements}

This research was supported by the Basic Science Research Program through the National Research Foundation of Korea (NRF) funded by the Ministry of Education, Republic of Korea (grant no. NRF-2017R1D1A3A03000532).

\section{Authors' Contributions}

Conception and design: YOK. Acquisition of data: YOK, SAC. Analysis and interpretation of data: YOK, SAC. Drafting the article:YOK, SAC. Critical revision of the article: YOK. Final approval of the version to be published: YOK, SAC.

\section{References}

1. Pavone $P$, Striano $P$, Falsaperla $R$, Pavone $L$, Ruggieri M. Infantile spasms syndrome, West syndrome and related phenotypes: what we know in 2013. Brain Dev 2014;36:739-51.

2. Lemke JR, Riesch $E_{1}$ Scheurenbrand $T$, Schubach M, Wilhelm C, Steiner I, et al. Targeted next generation sequencing as a diagnostic tool in epileptic disorders. Epilepsia 2012;53:1387-98.

3. Ko A, Youn SE, Kim SH, Lee JS, Kim S, Choi JR, et al. Targeted gene panel and genotype-phenotype correlation in children with developmental and epileptic encephalopathy. Epilepsy Res 2018;141:48-55.

4. Mignot C, Moutard ML, Trouillard O, Gourfinkel-An I, Jacquette $A$, Arveiler $B$, et al. STXBP1-related encephalopathy presenting as infantile spasms and generalized tremor in three patients. Epilepsia 2011;52:1820-7.

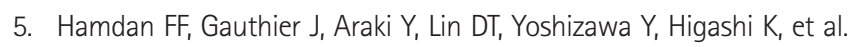
Excess of de novo deleterious mutations in genes associated with glutamatergic systems in nonsyndromic intellectual disability. Am J Hum Genet 2011;88:306-16.

6. Ohba C, Shiina M, Tohyama J, Haginoya K, Lerman-Sagie T, Okamoto $N$, et al. GRIN1 mutations cause encephalopathy with infantile-onset epilepsy, and hyperkinetic and stereotyped movement disorders. Epilepsia 2015;56:841-8.

7. Lemke JR, Geider $K$, Helbig KL, Heyne HO, Schütz H, Hentschel J, et al. Delineating the GRIN1 phenotypic spectrum: a distinct genetic NMDA receptor encephalopathy. Neurology 2016;86:2171-8.

8. Chen W, Yuan H. GRIN1 mutations in early-onset epileptic encephalopathy. Pediatr Neurol Briefs 2015;29:44.

9. Rossi M, Chatron N, Labalme A, Ville D, Carneiro M, Edery $P$, et al. Novel homozygous missense variant of GRIN1 in two sibs with intellectual disability and autistic features without epilepsy. Eur J Hum Genet 2017;25:376-80. 
10. Zehavi $Y$, Mandel H, Zehavi A, Rashid MA, Straussberg R, Jabur B, et al. De novo GRIN1 mutations: an emerging cause of severe early infantile encephalopathy. Eur J Med Genet 2017;60:317-20.

11. Fry AE, Fawcett $K A$, Zelnik N, Yuan $H$, Thompson BAN, Shemer-Meiri $L$, et al. De novo mutations in GRIN1 cause extensive bilateral polymicrogyria. Brain 2018;141:698-712.

12. Crino PB. Polymicrogyria and GRIN1 mutations: altered connections, altered excitability. Brain 2018;141:622-3.

13. Pironti E, Granata F, Cucinotta F, Gagliano A, Efthymiou S, Houlden $H_{\text {, }}$ et al. Electroclinical history of a five-year-old girl with GRIN1-related early-onset epileptic encephalopathy: a video-case study. Epileptic
Disord 2018;20:423-7.

14. Tang W, Liu D, Traynelis SF, Yuan H. Positive allosteric modulators that target NMDA receptors rectify loss-of-function GRIN variants associated with neurological and neuropsychiatric disorders. Neuropharmacology 2020;177:108247.

15. Intson $K$, van Eede MC, Islam R, Milenkovic M, Yan Y, Salahpour A, et al. Progressive neuroanatomical changes caused by Grin1 loss-offunction mutation. Neurobiol Dis 2019;132:104527.

16. Bosch DG, Boonstra FN, de Leeuw N, Pfundt R, Nillesen WM, de Ligt J, et al. Novel genetic causes for cerebral visual impairment. Eur J Hum Genet 2016;24:660-5. 